Published in final edited form as:

Neuroscience. 2008 October 15; 156(3): 597-606. doi:10.1016/j.neuroscience.2008.07.051.

\title{
The influence of reactivity of the electrode-brain interface on the crossing electric current in therapeutic deep brain stimulation
}

\author{
Nada Yousifa ${ }^{\mathrm{a}}$, Richard Bayford ${ }^{\mathrm{b}}$, and Xuguang Liu ${ }^{\mathrm{a}, \mathrm{c},{ }^{*}}$ \\ ${ }^{\text {aDepartment }}$ of Clinical Neuroscience, Division of Neuroscience and Mental Health, Faculty of \\ Medicine, Imperial College London \\ bThe Bio-modelling/Bio-informatics Group, Department of Natural Science, Institute of Social and \\ Health Research, Middlesex University, London \\ cThe Movement Disorders and Neurostimulation Unit, Department of Neuroscience, Charing \\ Cross Hospital, Imperial College Healthcare NHS Trust, London, UK
}

\begin{abstract}
The use of deep brain stimulation (DBS) as an effective clinical therapy for a number of neurological disorders has been greatly hindered by the lack of understanding of the mechanisms which underlie the observed clinical improvement in patients. This problem is confounded by the difficulty of investigating the neuronal effects of DBS in situ, and the impossibility of measuring the induced current in vivo. In our recent computational work using a quasi-static finite element (FEM) model we have quantitatively shown that the properties of the depth electrode brain interface (EBI) have a significant effect on the electric field induced in the brain volume surrounding the DBS electrode. In the present work, we explore the influence of the reactivity of the EBI on the crossing electric current using the Fourier-FEM approach to allow the investigation of waveform attenuation in the time domain. Results showed that the EBI affected the waveform shaping differently at different post-implantation stages, and that this in turn had implications on induced current distribution across the EBI. Furthermore, we investigated whether hypothetical waveforms, which were shown to have potential usefulness for neural stimulation but are not yet applied clinically, would have any advantage over the currently used square pulse. In conclusion, the influence of reactivity of the EBI on the crossing stimulation current in therapeutic DBS is significant, and affects the predictive estimation of current distribution around the implanted DBS electrode in the human brain.
\end{abstract}

\section{Keywords}

finite element model; computational simulation; tissue impedance

\section{Introduction}

The clinical therapy of deep brain stimulation (DBS) involves electrical stimulation via electrodes chronically implanted into selected brain targets in order to alleviate the symptoms of a number of movement and other neurological disorders (Lozano et al., 2002; Wichmann and DeLong, 2006; Kringelbach et al., 2007). This treatment evolved from ablative procedures when it was observed that such stimulation could provide a reversible alternative to lesioning the targeted nucleus (Benabid et al., 1987), and for this reason there

\footnotetext{
*Corresponding author: Dr Xuguang Liu, 10 $0^{\text {th }}$ Floor, East Block, Charing Cross Campus, Imperial College London, St Dunstan's Road, London W6 8RP, UK; x.liu@ic.ac.uk; Telephone: +44-208 8467631; Fax: +44-208 $3830663 .$.
} 
was a lack of systematic investigation into how the extracellular stimulation achieves the therapeutic outcomes. One of the key issues in the DBS treatment is that once the electrode is surgically implanted into the deep brain structure, a depth electrode-brain interface (EBI) is created, consisting of three basic structural elements: the quadripolar electrode, the perielectrode space and the surrounding brain tissue. It was demonstrated by physiological data that at the acute stage immediately post-implantation, the peri-electrode space is filled by extracellular fluid, and is modulated by physiological brain pulsation (Xie et al., 2006; Priori et al., 2006). Furthermore, electron microscopy (Moss et al., 2004) of explanted DBS electrodes revealed that after a few weeks of implantation, reactive growth of low conductivity giant cells occurs in the peri-electrode space and attaches to the surface of the explanted electrode. These changing biophysical properties of the peri-electrode space are common features of the depth EBI (van Kuyck et al., 2007) as they are independent from the brain regions the electrodes are implanted into, and from the disorders the electrodes are implanted for.

It has been understood for many years that the electrical properties of neural tissue have both a resistive and a reactive component (Cole and Curtis, 1936). The nature of this bioimpedance has been well studied and described experimentally (Pethig, 1987). This is because it is important to understand the impact that such properties have, on both electrical measurements when currents of interest travel across the EBI from neural tissues to an electrode, and on electrical stimulation when a stimulating current passes across the EBI into the tissue from an electrode. In both stimulation and recording, we expect that the resistive and reactive components of the EBI will influence the current crossing the interface in a frequency-dependent manner. Such biophysical characteristics of tissue have previously been modelled by an equivalent electrical circuit (McAdams and Jossinet, 1995) containing a capacitor and a resistor in parallel, which allows further understanding of the dynamic nature of the electrical properties of neural tissue. Considering the case for DBS specifically, reactive components may arise due to a number of parts of the stimulation system. While the implanted hardware such as the extension cable and the electrode body contribute constant and small values of resistance, the tissue impedance and the impedance at the EBI can vary due to the location of the electrode, the patient, or the duration of the implantation (Hemm et al., 2004). Furthermore, our recent results of depth recordings of local field potentials via the implanted DBS electrode revealed that the physiological modulation of the EBI in the acute stage via brain pulsation selectively affected the crossing neural signals in a frequencydependent manner, as the amplitude of the electrode potential was inversely correlated with that of the tremor-related oscillation of $4-6 \mathrm{~Hz}$, but not the beta oscillation of $15-20 \mathrm{~Hz}$ related to Parkinson's disease (Yousif et al., 2008).

Using a computational model of the 3-dimensional interface (Yousif et al., 2007; Yousif and Liu, 2007), we have shown that the EBI influences the induced electric field by shunting the current in the acute stage, and shielding the tissue from the stimulus at the chronic stage so that there is a need to top up the stimulating amplitude (Yousif et al., 2007; Yousif et al., 2008). This change over time of the biophysical properties of the interface should be accounted for in order to make more accurate predictions based on such models. However, solving the static Laplace equation with frequency independent conductivity parameters means that the model encompassed simplifying assumptions and did not account for the time dependence of the stimulus waveform in a significantly reactive tissue. A different approach has been presented for extending such quasi-static FEM models to account for the complex (frequency-dependent) conductivity of the tissue and the electrode, and combining these complex FEM models with Fourier analysis to obtain an estimate of the effect of time dependence of the waveform (Butson and McIntyre, 2005). This previous work has shown that the tissue and the electrode capacitance impact on the induced waveform significantly and differently depending on whether voltage-controlled or current-controlled stimulation is 
used. The understanding of the waveform induced in the tissue is an important extension for FEM models as the clinical outcome is known to be critically dependent on the waveform parameters (Kuncel and Grill, 2004), therefore changes in amplitude and shape which come about due to the electrical properties of the interface must be accounted for to reveal the true nature of the impact of the interface on the current crossing into the human brain.

We hypothesised that the reactivity of the peri-electrode space varies from being filled with extracellular fluid immediately post-implantation to giant cells at the chronic stage a few months post-implantation, which in turn would affect the voltage waveform of the electric pulses differently. The objectives of the present study focus on the effects of the reactive properties of the EBI, and its effects on the stimulation-induced waveform using the FourierFEM approach. We intend to demonstrate that the square pulse is altered in both amplitude and shape by the EBI at different stages post-implantation when the peri-electrode space is filled with either extracellular fluid of low resistance and low reactivity or high resistance and high reactivity giant cells, to investigate whether the square pulse shape also changes with distance from the electrode, and to estimate the effects of such distortion of the pulse waveform on the spatial distribution of the electric current in the surrounding brain tissue. Furthermore, we analyse these results in terms of the electrical behaviour of the interface using an equivalent circuit model of the EBI, which assists us to understand the contribution of each component to the behaviour observed. Finally, we extend the study to some clinically unused novel waveforms, which were recently proposed in a computational modelling study to be useful stimulation waveforms for DBS (Sahin and Tie, 2007) to investigate whether such waveforms, which have a different frequency profile to square pulses, are also distorted through the electrode-brain interface, and consequently whether they may prove to be more useful for neuromodulation. This combined approach allows us to consider the spatial spread of the induced electric field in the volume of neural tissue surrounding the implanted electrode, the shape of the potential induced in the tissue relative to the input waveform, as well as probing this behaviour in terms of the changing biophysical properties of the EBI. This in turn provides a useful tool for the visualisation of stimulation effects, and the possibility to make more accurate testable predictions about the evolving electrical behaviour of the interface.

\section{Experimental procedures}

In order to study the frequency-dependent reactive properties of the electrode brain interface during DBS, we combine our previous EBI model of a complex FEM approach with a Fourier-FEM method.

\section{The finite element model of the electrode-brain interface}

A detailed description of the structural EBI model was given previously (Yousif et al., 2007; Yousif and Liu, 2007), and is summarised here: We used COMSOL Multiphysics 3.3 (COMSOL AB, Stockholm, Sweden) to form the EBI model. The geometry of the EBI is defined by the description of the Medtronic 3389 electrode which has four $1.5 \mathrm{~mm}$ long platinum iridium contacts with three $0.5 \mathrm{~mm}$ long separations on a $1.23 \mathrm{~mm}$ diameter lead (Medtronic Inc, Minneapolis, MN, USA). A peri-electrode space is defined with a thickness of $0.25 \mathrm{~mm}$ separating the electrode from the surrounding brain tissue, which extends $10 \mathrm{~mm}$ away from the electrode (Figure 1). This thickness was selected to be wide enough to allow the FEM mesh in this layer to contain up to four elements and ensure the accuracy of the solution, while remaining small compared to the dimensions of the electrode and surrounding tissue. The model was meshed into tetrahedral elements using a Delaunay meshing algorithm (COMSOL AB, Stockholm, Sweden). 
The potential distribution induced via such an implanted electrode was calculated by solving Laplace's equation:

$$
\nabla \cdot \sigma^{*} \nabla V=0
$$

where $\nabla$ is the del operator and denotes the derivative of $V$ in each of the $(\mathrm{x}, \mathrm{y}, \mathrm{z})$ directions, $V$ is the scalar potential (measured in Volts), ' ' denotes a dot product.

In this study, we chose to move to a 2-dimensional representation in order to significantly reduce the simulation time, after having ensured that both the 3-dimensional and 2dimensional geometries gave the same results.

\section{The complex FEM approach}

Unlike previous work, in this study we utilise a complex version of the EBI model, in order to be able to investigate the frequency-dependent features of the electrode-brain interface. In the complex Laplace equation $\sigma^{*}=\sigma-i \omega \varepsilon_{0} \varepsilon_{r}, \sigma$ is the conductivity (measured in Siemens per metre), $i$ is the imaginary unit, or $\sqrt{-1}, \omega$ is the angular frequency or $2 \pi \mathrm{f}$, where $\mathrm{f}$ is the frequency, $\varepsilon_{O}$ is a constant known as the permittivity of free space $\left(8.85 \times 10^{-12}\right.$ Farads per metre), and $\varepsilon_{r}$ is the relative permittivity, which is a material specific parameter.

As the potential distribution between different EBI conditions was our primary focus, the anatomy of the surrounding brain tissue is simplified to homogenous grey matter with conductivity as $\sigma=0.2 \mathrm{~S} / \mathrm{m}$ (Geddes and Baker, 1967), and we used a maximum and a minimum (measured at low frequencies $10-1000 \mathrm{~Hz}$ ) for the relative permittivity (relative to free space): $1 \times 10^{7}$ and $1 \times 10^{6}$ (http://niremf.ifac.cnr.it/tissprop/). To simulate the EBI at the acute stage, during which the peri-electrode space is filled by extracellular fluid, a value of $\sigma=1.7 \mathrm{~S} / \mathrm{m}$ (Rabbat, 1990) and $\varepsilon_{r}=109$ was used (http://niremf.ifac.cnr.it/tissprop/). At the chronic stage, pathological growth of giant cells covers the electrode surface (Moss et al., 2004), and this tissue has been shown to be largely fibrous in nature (Haberler et al., 2000; Henderson et al., 2002; Nielsen et al., 2007). In order to model this, the conductivity and permittivity values were set equivalent to white matter such that $\sigma=0.125 \mathrm{~S} / \mathrm{m}$, and $\varepsilon_{r}=$ $1 \times 10^{7}$ (maximum), $1 \times 10^{6}$ (minimum) (http://niremf.ifac.cnr.it/tissprop/). The conductivity and permittivity of the electrode surface were as follows: For the platinum/ iridium contacts $\sigma=5 \times 10^{6} \mathrm{~S} / \mathrm{m}$ (www.eddy-current.com/condres.htm), and $\varepsilon_{\mathrm{r}}=1$, and for the urethane shaft $\sigma=1 \times 10^{-13} \mathrm{~S} / \mathrm{m}$ and $\varepsilon_{\mathrm{r}}=2$ and were estimated based on measurements of similar urethane polymers (Krol et al., 2006).

In all simulations the amplitude of the stimulating potential via the active contact was set to a selected value $(-1 \mathrm{~V})$, and the outer boundary of the surrounding tissue $(10 \mathrm{~mm}$ away from the surface of the electrode) was set to $0 \mathrm{~V}$, both via Dirichlet boundary conditions. The nonactive contacts and the electrode shaft were bound using Neumann conditions to simulate inactivated contacts and the insulated shaft.

\section{The Fourier-FEM approach}

This approach was previously described (Butson and McIntyre, 2005) to provide a method to estimate the time-dependent effects of stimulation on the electric field induced in the surrounding neural tissue. It involves the following steps: First the stimulus waveform, here assumed to be a perfect monophasic square wave (Figure 2a), is constructed in the time domain (Matlab, Mathworks). Secondly, this waveform is transformed into the frequency domain using a 1024 point discrete-Fourier transform (DFT). Next, the complex finite element model is solved at 513 frequencies. The complex electric potential calculated from the FEM model at each frequency is then scaled by the amplitude of the DFT (Figure 2b) 
and shifted by the phase of the DFT (Figure 2c). Finally the waveform is transformed back into the time domain using an inverse DFT (Figure 2d). This process is schematised in Figure 2. Specifically, we used a time range of 0 to $1 \mathrm{~ms}$, and performed the DFT at 1024 steps. Therefore we solved the FEM model at 513 frequencies from $0 \mathrm{~Hz}$ to $512 \mathrm{kHz}$. We ran our simulations using this number of time steps and value of dt, and we also ran simulations using the parameters used by Butson and McIntyre (Butson and McIntyre, 2005) in order to ensure that there were no discrepancies due to different selections of parameters. In this case, the number of time steps was also 1024, but dt was set at 1e-5s so that the waveform in the time domain was defined between 0 and $0.01024 \mathrm{~s}$, and the frequency range was from 0 to $50 \mathrm{kHz}$. As expected, we found that this change in parameters made no qualitative difference to the results presented below.

\section{Novel waveforms}

Recently, waveforms other than the ideal square wave which is currently used for DBS have been proposed as providing a better alternative for neurostimulation (Sahin and Tie, 2007). Using the Fourier-FEM model, we investigated three of the waveforms which were shown to perform as well or better than a square wave: a linear decrease; an exponential decrease; and a Gaussian waveform. The potential induced in the tissue by each of these waveforms was simulated in both acute and chronic conditions, and the waveforms were compared relative to the square pulse in terms of the maximum induced potential amplitude achieved.

\section{Quantification of the induced potential waveforms over distance and over time}

The potential waveform induced in the surrounding neural tissue at any time point and at any measuring position may potentially be quantified. The decay in the waveform amplitude over distance was measured at distance increments of $0.5 \mathrm{~mm}$ from the surface of the electrode contact 0 to the brain volume boundary of a maximum distance $10 \mathrm{~mm}$ (Figure 4(c)); and to assess the potential induced in the neural tissue over time, comparison is made between measurements obtained at $0.5 \mathrm{~mm}$ away from the surface of the electrode contact 0 . We compared these waveforms by measuring the peak amplitude, and by measuring the area under the voltage waveform which gives a measure of the energy delivered to the tissue. We then compared across conditions by calculating a simple percentage as value2/value $1 *$ $100 \%$. It is important to note that we do not claim that our simulation results yield absolute values for the induced potential distribution in the surrounding tissue by DBS, but use this comparison across simulations to look at the relative differences between time stages and waveforms. This relative comparison should eliminate concern over absolute errors in estimation of the effects of DBS.

\section{Circuit modelling}

While using the Fourier FEM model allows us to probe the structural properties of the interface on the distortion in the waveform, in order to understand the results in more detail, we used PSPICE (Cadence, San Jose, CA, USA) to model an equivalent electric circuit of the EBI, therefore allowing us to investigate the specific contribution of each electrical part of the interface in more detail. This circuit is based upon previous studies of the electrodeelectrolyte interface, which represent the interface as an RC circuit (Geddes, 1972). If we assume that the electrode is perfectly polarisable, and therefore no electrons cross the interface, which is reasonable assumption for a platinum electrode, then the electrode properties are dominated by capacitance, and can be modelled as a capacitor (Butson and McIntyre, 2005). In addition, each additional compartment of our EBI model, the PES and the surrounding neural tissue is represented by a RC pair (Neuman, 1997), mimicking the FEM model in which we have conductivity and permittivity. Therefore the circuit consisted of a square pulse voltage source in series with a capacitor representing the electrodeelectrolyte interface, which in turn is in series with an RC pair for the peri-electrode space, 
and the tissue, as shown in Figure 3. Parameters for each of the components in this model were calculated based on the following. For a metal in an aqueous solution, it was previously reported that the electrode capacitance is in the range $10-20 \mu \mathrm{F} / \mathrm{cm}^{2}$ (Merrill et al., 2005), and therefore for a DBS contact, which has a surface area of $0.06 \mathrm{~cm}^{2}$, the double layer capacitance is set at $0.6 \mu \mathrm{F}$ to $1.2 \mu \mathrm{F}$. For the peri-electrode space, we use the following equations to calculate $\mathrm{C}$ and $\mathrm{R}$ based on the conductivity and permittivity parameters given above:

$$
C=\frac{\varepsilon_{0} \varepsilon_{r} A}{l} \text { and } R=\frac{\rho l}{A}
$$

where $A$ is the area of the contact, I represents the thickness of the peri-electrode space (PES), and $\rho$ is the resistivity which is the inverse of conductivity, and all other symbols are as described above. These equations lead to values of $C_{\text {acute }}=2.3 \times 10^{-11} \mathrm{~F}, R_{\text {acute }}=25 \Omega$, $C_{\text {chronic }}=2.1 \times 10^{-6} \mathrm{~F}$, and $R_{\text {chronic }}=334 \Omega$.

Finally for the bulk tissue resistance we used the following equation (Weinman and Mahler, 1964):

$$
R=\frac{1}{2 \pi \sigma a p}
$$

where $a$ is the radius of the electrode and $p$ is a shape factor, to account for the derivation of this equation based on hemi-spherical electrodes. Using $p=0.85$, we obtain $R_{\text {tissue }}=1480 \Omega$.

Based on the time constant $\left(\tau=\frac{\varepsilon_{0} \varepsilon_{r}}{\sigma}=R C\right)$ we could then calculate $C_{\text {tissue }}=2.99 \times 10^{-7} \mathrm{~F}$.

\section{Results}

\section{The impact of the electrode brain interface on the conventional square pulses}

The initial simulations we ran look at the waveform resulting in the tissue in two conditions, in the acute time stage with fluid surrounding the newly implanted electrode, and the chronic time stage when the electrode is surrounded by encapsulation tissue.

In the acute stage, the waveform is no longer a perfect square wave (Figure 4), but has a slowly rising phase, which plateaus if the pulse width is long enough, and then exponentially falls at the end of the step. This is typical of the low-pass filter behaviour of an RC circuit, and we found that this effect is more pronounced with the higher tissue permittivity parameters.

In the chronic stage, as in the quasi-static model, the overall amplitude is smaller than in the acute stage, but the shaping of the waveform is very different. The potential peaks quickly as the stimulating square pulse does with an infinitely small rise time, and then the amplitude decreases over the pulse width. Finally, the voltage drops with an infinitely small time step, and drops to $-6 \mathrm{mV}$ before rising slowly to zero. Once more, this effect is more marked at the higher value of tissue permittivities.

In both cases, the shaping of the waveform remained constant with increasing distance from the electrode contact. We then looked at the drop-off in the peak amplitude of the induced waveform with increasing distance from the electrode contact (line of measurement shown in Figure 4(c)). The rate of decrease in the potential is shown by examination of Figure 4(d) (solid lines) and is more marked for the chronic case, which reflects the overall reduced 
amplitude of potential in the chronic case. However this difference between acute and chronic cases is $10 \%$, which is less than the difference calculated based on a quasi-static model (equivalent to the solution of the Fourier-FEM model at $0 \mathrm{~Hz}$ ), which is $19 \%$ (Figure 4(d) dashed lines). This indicates that the differences between potential spread in these two cases may be less than previously shown. In the acute stage, the energy delivered to the tissue, as estimated by the area under the induced waveform, is $93 \%$ of the original waveform, and in the chronic case this drops to $76 \%$, indicating a change of $17 \%$ as a result of encapsulation.

We also looked at the effect of increasing the pulse width of the original waveform (Figure 5 ) in terms of how this influences the induced waveform. The shape observed with the $60 \mu \mathrm{s}$ pulse-width was maintained with all other pulse-widths examined, with small changes in the maximum amplitude achieved (1-2\%), and no change in the energy delivered relative to the original waveform.

\section{Comparing the conventional square pulses with novel waveforms}

We also investigated and compared three novel waveforms to the conventional square pulses, which were recently proposed to be a useful to stimulate neural tissue based on computational modelling. Figure 6 shows that these waveforms appear to be less influenced by the interface model than the square wave in both the acute and the chronic time stages. In each case, the energy delivered by the induced waeforms relative to the original stimulus (dashed lines in Figure 6) is unchanged across all stimuli, because each result is based on the FEM solutions at the same component frequencies. However, we compared the energy delivered by each waveform in acute and chronic stages relative to the original waveform (Table). This clearly shows that the novel waveforms will deliver much less energy than a square wave, and therefore prolong battery life. Also, as listed in the Table, the maximum amplitude attained does change in the acute case across the different waveforms, while in the chronic case they remain stable relative to the input. In the acute case, the square wave and the Gaussian wave achieve the highest amplitude potential in the tissue, with the two decreasing waveforms attaining less potential by approximately $10 \%$ in the linear decrease case and $20 \%$ in the exponential decrease case. Finally, the simulation of the Gaussian waveform shows that there is a phase shift of the waveform in the acute case, which is not present in the chronic case.

\section{The waveform shaping in an equivalent circuit model}

In order to understand these Fourier-FEM results in relation to the electrical properties of the interface, we used an equivalent circuit model of the EBI (Fig. 3). This model includes a capacitance and resistance in parallel for the PES and tissue compartments, while the electrode is modelled as a pure capacitor. We model the change over time which affects the electrical properties of the EBI from the acute to the chronic stage, by changing the resistance and the conductivity of the peri-electrode space compartment of the circuit as described above, which is comparable to changing the conductivity and permittivity in the FEM model. Figure 7 shows that this model displays the same shaping of the square wave in the tissue as the FEM model does (Fig. 4(A)), which indicates that the equivalent circuit captures the relevant electrical features of the EBI which cause a distortion of the input waveform.

\section{Discussion}

The present study extends our previous investigations on the influence of the resistive component of the EBI using a quasi-static FEM model. In particular we further investigate the reactive properties of the EBI, and its effects on the stimulation-induced waveform in the 
surrounding brain volume using the Fourier-FEM approach. The main findings of the present study are: 1) there are frequency-dependent effects of the EBI, which result in dramatic waveform distortions which depend on the different compositions of the perielectrode space in the acute and chronic time stages post-implantation; 2) these distorted electric potentials can be quantified over time at one position or over distance at the same time point; 3) the use of stimulus waveforms with different frequency content may change the EBI distortion, and consequently may result in better predicted stimulation effects. The present combined modelling approach provides a useful tool for the visualisation of stimulation-induced current, and the possibility to make more accurate testable predictions about the evolving electrical behaviour of the interface.

Our results highlight a number of issues related to the EBI, which we discuss here. First, we observed that the structurally homogenous models of the EBI in which the peri-electrode space is not defined as an independent structural component exhibited little frequencydependent behaviour in affecting the crossing current. This is consistent with the results of a theoretical model of electric field effects in homogenous neural tissue (Bedard et al., 2004) which showed that in a medium with constant conductivity and permittivity, as in the homogenous EBI model, there is no frequency-dependent influence on the extracellular potential. The use of homogenous models was also previously shown to be inadequate for estimating the stimulation effects of DBS (Butson and McIntyre, 2005), and it was suggested by pathological (Moss et al., 2004), neurophysiological (Xie et al., 2006) and computational modelling (Yousif et al., 2007) studies that the peri-electrode space is crucial for determining the electric current injected via the electrode to the brain tissue across the EBI. Here we have shown that it is the relative dominance of the tissue capacitance compared to the capacitance of the peri-electrode space, which change post-implantation from extracellular fluid of high conductivity and low permittivity to the reactive giant cells of high impedance and permittivity, which influences the waveform shaping. Second, it is crucial to be able to accurately estimate the potential in the surrounding brain volume induced by the injected current in which both the time dependent and non-time dependent components are considered. This is important for using such estimates to understand how the stimulating potential interacts with neurons or neural fibres to modulate their activity. In the present work, we have shown that the quasi-static model over estimates the induced potential amplitude compared to the Fourier FEM approach by as much as 9\% when comparing the cellular peri-electrode space at the chronic stage and the fluid peri-electrode space of low capacitance. Interestingly, this is lower than another study (Butson and McIntyre, 2005), but in that case they compared the effects of stimulation on neural fibres and found that the quasi-static models overestimated the volume of tissue activated by $20 \%$ compared to the Fourier-FEM approach. Third, this matter may be complicated even further when the EBI in the transient stage of post-implantation is considered, during which it was shown in previous studies (van Kuyck et al., 2007) and our recent study (Yousif et al., 2008) that both the biophysical properties of the medium in the peri-electrode space and the pattern of capsulation around the electrode surface vary with infinite possibilities so that the shielding effect of partial giant cell growth on the injected current could shape the induced electric potential in an unpredictable manner.

Compared to our previous simulations on the amplitude difference between stimulation in the acute stage, where the induced extracellular potential in the brain volume surrounding the electrode is stronger than that induced at chronic stages, due to the higher conductivity of extra-cellular fluid than of encapsulation tissue (Yousif et al., 2007; Yousif et al., 2008), we have demonstrated in the present study that there is also a change in the characteristics of the reactive component of the interface, which impacts on the waveform shaping in the two clinical time stages. In the acute stage, the shaping is most significant at the start and at the end of the square pulse, mimicking typical low-pass filter behaviour. Whereas, in the 
chronic stage, the rise and fall times of the potential passed through the EBI are similar to the original waveform, while at the DC or plateau stage, the amplitude of the potential does not remain constant as in the original stimulus, but decays over time. It should be noted that in the chronic stage the main effect of the EBI is an overall decrease in amplitude of the potential waveform, the distortion in waveform shape being of comparatively minor consequence. Interestingly, the pulse-width does not alter these specific features of waveform distortion. The equivalent circuit model replicated the results of the Fourier-FEM approach very well (Figure 4(a) and Figure 7), which is because similar electrical properties were represented in the two models. The circuit model allowed us to show that this specific difference in behaviour of waveform attenuation at the two post-implantation stages can be attributed to the relative dominance of the tissue capacitance compared to the capacitance of the peri-electrode space rather than the absolute capacitance of the peri-electrode space alone. In the acute stage the tissue capacitance is much greater (four orders of magnitude) than the capacitance of the fluid filled layer, and consequently we see the typical capacitive effect on the rise time of the voltage pulse in the tissue. However, in the chronic stage when the encapsulation capacitance is an order of magnitude greater that that of the tissue, the change in the voltage waveform is greater at the interface compared to at the tissue. Therefore the relative capacitance of the peri-electrode space and the tissue has a major effect on determining the stimulation induced waveform properties in the neural tissue. A similar mechanism may also apply to the relative dominance of the electrode capacitance compared to the capacitance of the peri-electrode space at different stages (Butson and McIntyre, 2006).

Such waveform shaping at the different post implantation stages may be quite significant for both depth stimulation and depth recording. First, it has been previously observed that the EBI may filter recorded local field potentials in a frequency dependent manner (Yousif et al., 2008), and this property can also be seen in the low-pass nature of the acute EBI. This in turn may have an impact on depth recordings, which are made in the acute stage when the electrodes are implanted but externalised. Second, in terms of stimulation these different waveforms may have different effects on surrounding neurons. We can assess this effect by looking at the activation function (Rattay, 1989) which shows that during most of the waveform the acute interface generates higher magnitudes of activation function. The exceptions are at the start and end of the pulses when the sharp transients in the chronic waveform lead to the activation function having a higher magnitude at these points. This indicates that the timing of neuronal activation may vary slightly over the duration of a stimulus pulse in the different stages post implantation.

One of the objectives of the present study is to utilise the present complex EBI model to investigate whether different stimulation waveforms, which have a different frequency content from the conventional and clinical applicable square pulses and have been proposed to be effective at stimulating neurons (Sahin and Tie, 2007), so that the effects of DBS may be optimised by uncovering better alternatives of stimulation waveform to the conventional square pulses. Given that we have shown that the biophysical characteristics of the EBI changes dramatically over time post-implantation, the use of waveforms with simpler frequency spectra than that of the square pulses may be less distorted over the different time stages post-implantation. We studied three waveforms: a pulse with a linear decrease, a pulse with an exponential decrease, and a Gaussian waveform. Qualitatively, the resulting waveforms appear to be less attenuated by the EBI and the difference between the shape of the waveform at acute and chronic stages also seem less marked, though the amplitude difference between acute and chronic waveforms did not seem to be improved by using such stimuli (Table), which is expected, as it is largely a result of the resistive rather than capacitive property of the interface. Interestingly, the simulation with the Gaussian input 
revealed a phase shift in the acute stage, once more suggesting that the capacitive properties of the tissue may be more significant at the acute interface.

The work described here attempts to generate testable hypotheses about the effects of neurostimulation based on the estimated simulation of current distribution and the volume of tissue activated (VTA) around the implanted DBS electrode, which can be verified and ultimately applied to optimise clinical outcome. However, as with all modelling studies, this approach encompasses a number of necessary simplifications and assumptions, therefore it is crucial to discuses shortcomings and to ensure the reliability of the simulation results generated by a given model. With respect to the present FEM model, we set two parameters per structural compartment of the model, the conductivity and the permittivity. By doing so, if there are any discrepancies in the values used, we are able overcome this by looking at relative differences in the potential distribution induced in the tissue, and not claiming that our results provide absolute quantities. Several issues should be considered in future work: First, the current FEM model does not contain any information about the electrode itself, as the electrode surface is taken to be a boundary so material information is neglected. Future work should endeavour to categorise the contact impedance at the EBI (Cantrell et al., 2008). Second, a recent study (Bossetti et al., 2008) showed that the Fourier-FEM approach does not account for the frequency-dependence of the conductivity and permittivity parameters, nor for the inductive or propagation properties of neural tissue. This results in an error compared to the solution of the Helmholtz equation, which should also be considered in future work. In the circuit modelling, we assumed that the electrode is perfectly polarisable and can be modelled as a capacitor. This has been shown to be a valid assumption for DBS electrodes (Butson and McIntyre, 2005; Merrill et al., 2005). However the inclusion of other electrical components at the EBI, such as the parallel electrode resistance, may introduce complexities to the behaviour seen here. Third, the implantable DBS system involves more hardware than our equivalent circuit model represents, such as the cabling of the DBS electrode lead, which contain additional resistances and capacitances, as well as capacitances between contacts. However, in this study we focus on monopolar stimulation, therefore only one contact need be represented, and the effects of stimulation local to the interface, where cabling effects on the waveform are ignored.

In conclusion, the study has shown that the waveform induced in the neural tissue by DBS changes relative to the input waveform, and that this distortion is also dependent upon the nature of the interface, such that at acute stages post-implantation, the tissue capacitance dominates inducing a low-pass filter type behaviour, and at chronic stages post-implantation, the capacitance of the encapsulation itself takes over, attenuating the waveform shape. These dynamic biophysical properties of the interface may call for different classes of waveforms to be used because waveforms with different frequency content appear to be less distorted in shape by the EBI. We believe that the present study provides a deeper understanding of the time-dependent effects of the EBI on the current crossing the interface. This approach may provide better insight into the interactions of DBS with pathological activity, by estimating and visualising the stimulation current more accurately, and consequently to assist optimisation of the therapeutic outcomes.

\section{Acknowledgments}

Nada Yousif was supported by a research fellowship (grant ID 78512) from the Medical Research Council of the UK.

\section{List of abbreviations}

DBS Deep brain stimulation 


$\begin{array}{ll}\text { DFT } & \text { Discrete Fourier transform } \\ \text { EBI } & \text { Electrode-brain interface } \\ \text { FEM } & \text { Finite element method } \\ \text { PES } & \text { Peri electrode space } \\ \text { VTA } & \text { Volume of tissue activated }\end{array}$

\section{REFERENCES}

Bedard C, Kroger H, Destexhe A. Modeling extracellular field potentials and the frequency-filtering properties of extracellular space. Biophys J. 2004; 86:1829-1842. [PubMed: 14990509]

Benabid AL, Pollak P, Louveau A, Henry S, de RJ. Combined (thalamotomy and stimulation) stereotactic surgery of the VIM thalamic nucleus for bilateral Parkinson disease. Appl Neurophysiol. 1987; 50:344-346. [PubMed: 3329873]

Bossetti CA, Birdno MJ, Grill WM. Analysis of the quasi-static approximation for calculating potentials generated by neural stimulation. J Neural Eng. 2008; 5:44-53. [PubMed: 18310810]

Butson CR, McIntyre CC. Tissue and electrode capacitance reduce neural activation volumes during deep brain stimulation. Clin Neurophysiol. 2005; 116:2490-2500. [PubMed: 16125463]

Butson CR, McIntyre CC. Role of electrode design on the volume of tissue activated during deep brain stimulation. J Neural Eng. 2006; 3:1-8. [PubMed: 16510937]

Cantrell DR, Inayat S, Taflove A, Ruoff RS, Troy JB. Incorporation of the electrode-electrolyte interface into finite-element models of metal microelectrodes. J Neural Eng. 2008; 5:54-67. [PubMed: 18310811]

Cole KS, Curtis HJ. Cold Spring Harbor Symposia on Quantitative Biology. 1936; 4:73-89.

Geddes, LA. Electrodes and the measurement of bioelectric events. Wiley; 1972.

Geddes LA, Baker LE. The specific resistance of biological material--a compendium of data for the biomedical engineer and physiologist. Med Biol Eng. 1967; 5:271-293. [PubMed: 6068939]

Haberler C, Alesch F, Mazal PR, Pilz P, Jellinger K, Pinter MM, Hainfellner JA, Budka H. No tissue damage by chronic deep brain stimulation in Parkinson's disease. Ann Neurol. 2000; 48:372-376. [PubMed: 10976644]

Hemm S, Vayssiere N, Mennessier G, Cif L, Zanka M, Ravel P, Frerebeau P, Coubes P. Evolution of Brain Impedance in Dystonic Patients Treated by GPi Electrical Stimulation. Neuromodulation. 2004; 7:67-75. [PubMed: 22151186]

Henderson JM, Pell M, O’Sullivan DJ, McCusker EA, Fung VS, Hedges P, Halliday GM. Postmortem analysis of bilateral subthalamic electrode implants in Parkinson's disease. Mov Disord. 2002; 17:133-137. [PubMed: 11835450]

Kringelbach ML, Jenkinson N, Green AL, Owen SL, Hansen PC, Cornelissen PL, Holliday IE, Stein J, Aziz TZ. Deep brain stimulation for chronic pain investigated with magnetoencephalography. Neuroreport. 2007; 18:223-228. [PubMed: 17314661]

Krol P, Krol B, Subocz L, Andruszkiewicz P. Polyurethane anionomers synthesised with aromatic, aliphatic or cycloaliphatic diisocyanates, polyoxyethylene glycol and 2,2-bis(hydroxymethyl)propionic acid. Part 3. Electrical properties of polyurethane coatings. Colloid and Polymer Science. 2006; 285:177-183.

Kuncel AM, Grill WM. Selection of stimulus parameters for deep brain stimulation. Clin Neurophysiol. 2004; 115:2431-2441. [PubMed: 15465430]

Lozano AM, Dostrovsky J, Chen R, Ashby P. Deep brain stimulation for Parkinson's disease: disrupting the disruption. Lancet Neurol. 2002; 1:225-231. [PubMed: 12849455]

McAdams ET, Jossinet J. Tissue impedance: a historical overview. Physiol Meas. 1995; 16:A1-13. [PubMed: 8528108]

Merrill DR, Bikson M, Jefferys JG. Electrical stimulation of excitable tissue: design of efficacious and safe protocols. J Neurosci Methods. 2005; 141:171-198. [PubMed: 15661300] 
Moss J, Ryder T, Aziz TZ, Graeber MB, Bain PG. Electron microscopy of tissue adherent to explanted electrodes in dystonia and Parkinson's disease. Brain. 2004; 127:2755-2763. [PubMed: 15329356]

Neuman, MR. Biopotential electrodes. In: Webster, JG., editor. Medical Instrumentation: application and design. 3rd Edition. Wiley; 1997. p. 183-232.

Nielsen MS, Bjarkam CR, Sorensen JC, Bojsen-Moller M, Sunde NA, Ostergaard K. Chronic subthalamic high-frequency deep brain stimulation in Parkinson's disease--a histopathological study. Eur J Neurol. 2007; 14:132-138. [PubMed: 17250719]

Pethig R. Dielectric properties of body tissues. Clin Phys Physiol Meas. 1987; 8(Suppl A):5-12. [PubMed: 3568571]

Priori A, Ardolino G, Marceglia S, Mrakic-Sposta S, Locatelli M, Tamma F, Rossi L, Foffani G. Lowfrequency subthalamic oscillations increase after deep brain stimulation in Parkinson's disease. Brain Res Bull. 2006; 71:149-154. [PubMed: 17113940]

Rabbat, A. Tissue Resistivity. In: Webster, JG., editor. Electrical Impedance Tomography. Gallard Printers Ltd.; Bristol, London: 1990. p. 8-20.

Rattay F. Analysis of models for extracellular fiber stimulation. IEEE Trans Biomed Eng. 1989; 36:676-682. [PubMed: 2744791]

Sahin M, Tie Y. Non-rectangular waveforms for neural stimulation with practical electrodes. J Neural Eng. 2007; 4:227-233. [PubMed: 17873425]

van Kuyck K, Welkenhuysen M, Arckens L, Sciot R, Nuttin B. Histological Alterations Induced by Electrode Implantation and Electrical Stimulation in the Human Brain: A Review. Neuromodulation. 2007; 10:244-261. [PubMed: 22150838]

Weinman J, Mahler J. An analysis of electrical properties of metal electrodes. Med Electron Biol Eng. 1964; 33:310.

Wichmann T, DeLong MR. Deep brain stimulation for neurologic and neuropsychiatric disorders. Neuron. 2006; 52:197-204. [PubMed: 17015236]

Xie K, Wang S, Aziz TZ, Stein JF, Liu X. The physiologically modulated electrode potentials at the depth electrode-brain interface in humans. Neurosci Lett. 2006; 402:238-243. [PubMed: 16697525]

Yousif N, Bayford R, Bain PG, Liu X. The peri-electrode space is a significant element of the electrode-brain interface in deep brain stimulation: A computational study. Brain Res Bull. 2007; 74:361-368. [PubMed: 17845911]

Yousif N, Bayford R, Wang S, Liu X. Quantifying the effects of the electrode-brain interface on the crossing electric currents in deep brain recording and stimulation. Neuroscience. 2008; 152:683691. [PubMed: 18304747]

Yousif N, Liu X. Modeling the current distribution across the depth electrode-brain interface in deep brain stimulation. Expert Rev Med Devices. 2007; 4:623-631. [PubMed: 17850197] 


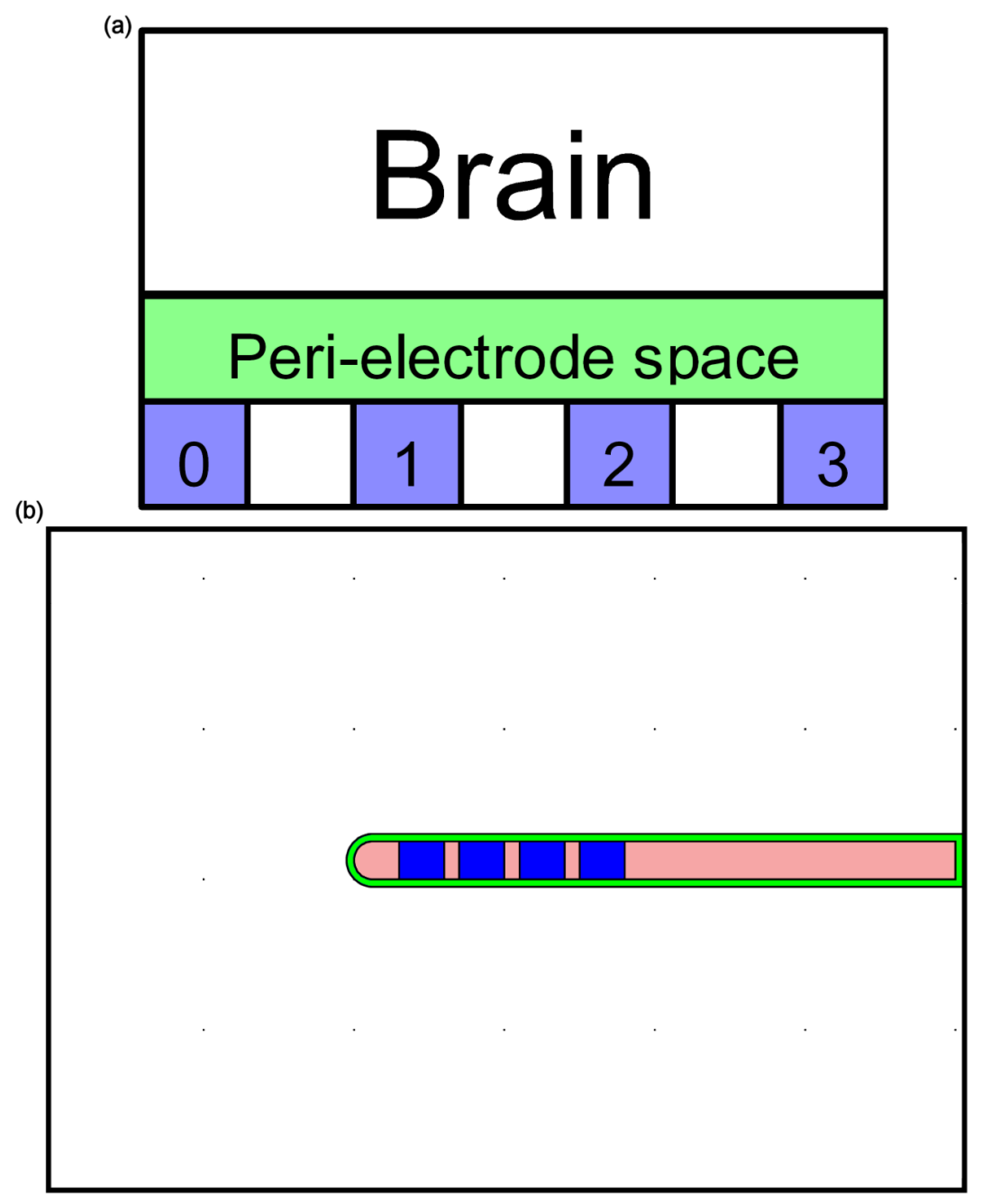

Figure 1.

(a) The EBI model consists of three compartments: The DBS electrode which consist of four contacts $(0,1,2$ and 3$)$; the peri-electrode space which changes from fluid-filled in the acute stage to a layer of encapsulation in the chronic stage; and the surrounding brain tissue. (b) The structure of the FEM model consists of the DBS electrode (Medtronic model 3389), which is uniformly surrounded by a peri-electrode space of $0.25 \mathrm{~mm}$ thickness, which in turn is surrounded by the neural tissue extending $10 \mathrm{~mm}$ from the electrode surface. 
(a)

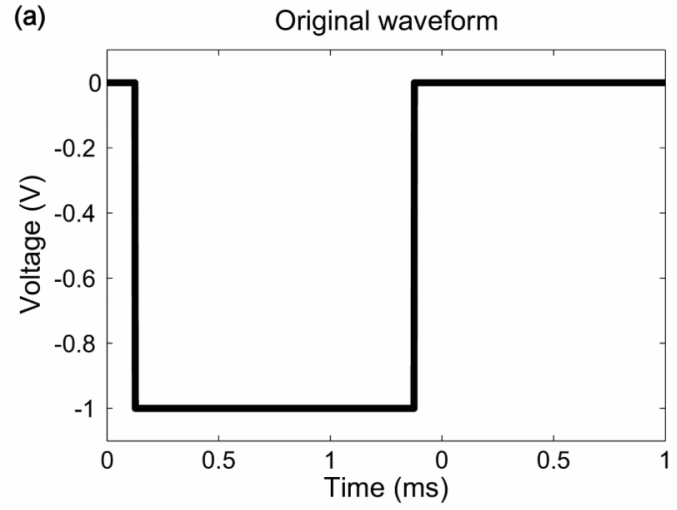

(b)

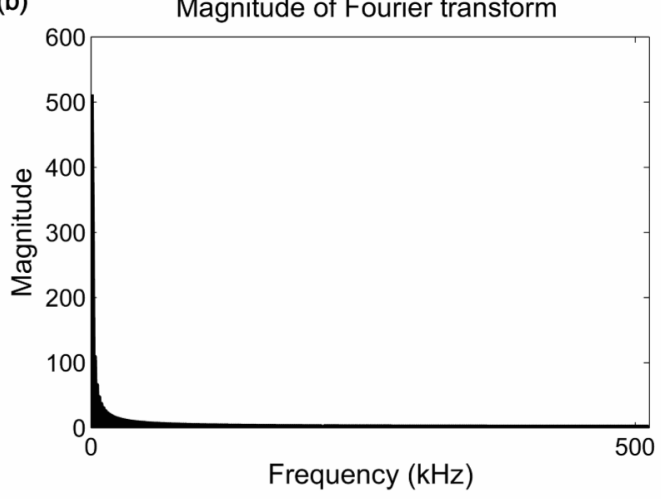

(d) After interface

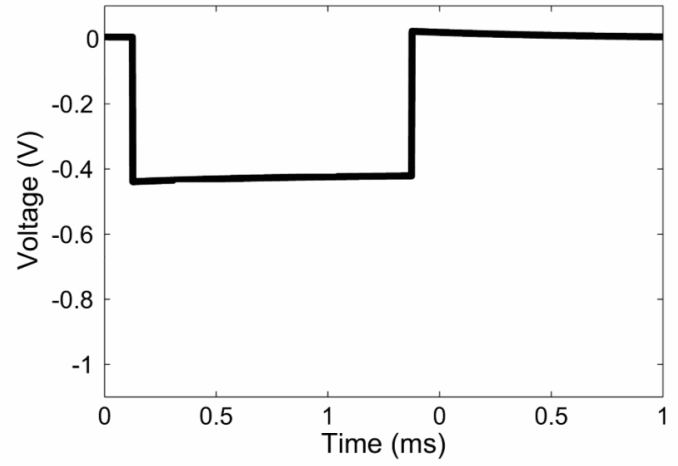

(c)

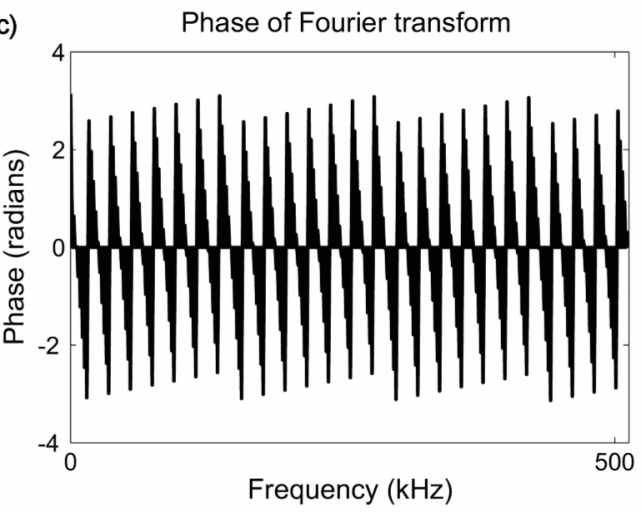

Figure 2.

A schematic diagram of the major steps of the Fourier-FEM approach: The original square pulse waveform is illustrated in the time domain (a), along with the amplitude (b) and phase (c) of its Fourier transform. The resulting waveform after distortion by the EBI is also shown (d) in the time domain. 


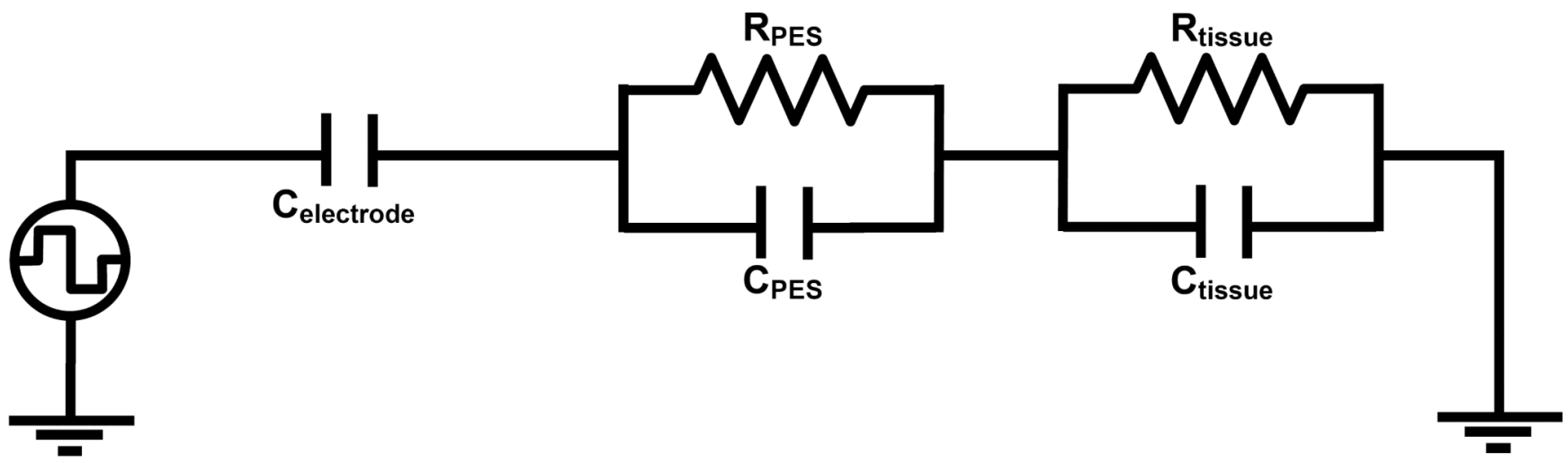

Figure 3.

The equivalent circuit model of the EBI was defined as consisting of a capacitor $\left(\mathrm{C}_{\text {electrode }}\right)$ to represent the non-polarisable platinum electrode, and an $\mathrm{RC}$ pair for the peri-electrode space ( $\mathrm{R}_{\mathrm{PES}}$ and $\left.\mathrm{C}_{\mathrm{PES}}\right)$, and another $\mathrm{RC}$ pair for the surrounding neural tissue $\left(\mathrm{R}_{\mathrm{tissue}}\right.$ and $\mathrm{C}_{\text {tissue }}$. 
(a)

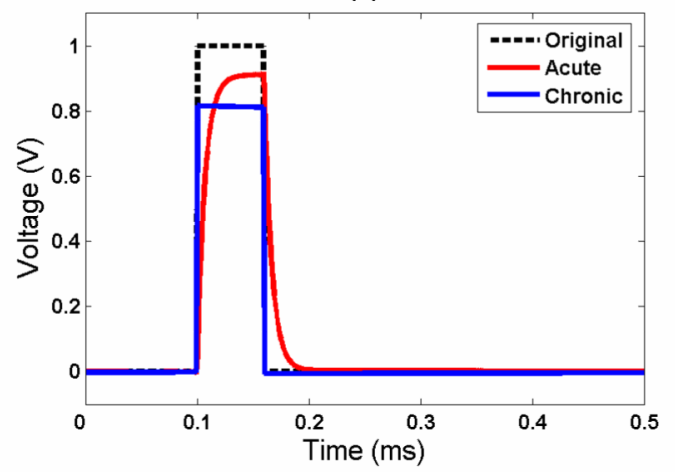

(c)

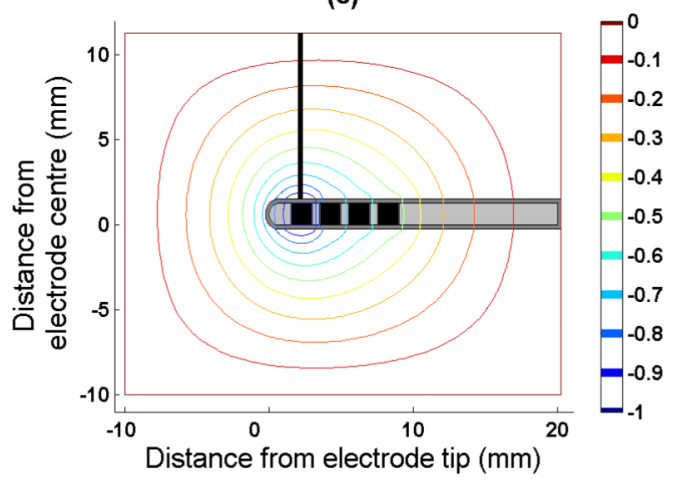

(b)

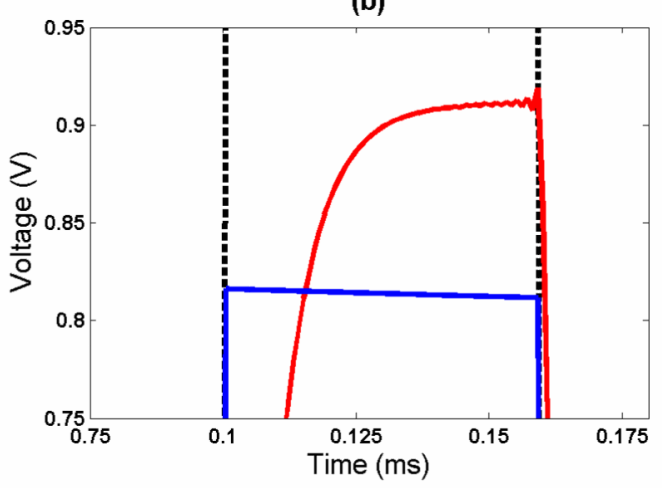

(d)

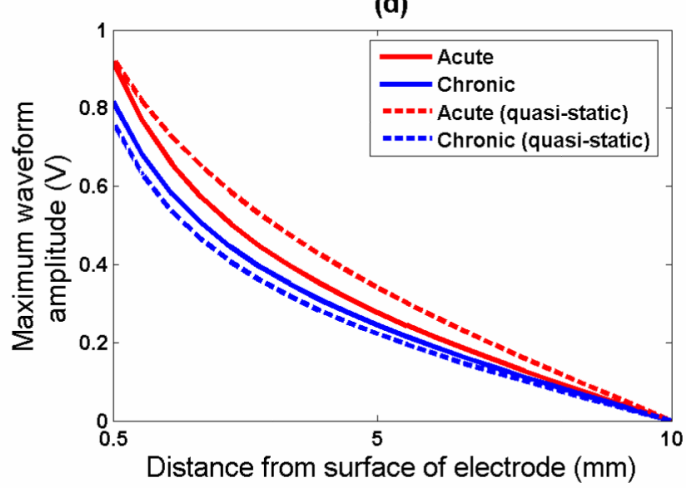

Figure 4.

This figure shows the effect of the interface on pulse-shaping. (a) The acute case displays slow rise and fall times, which is similar to the behaviour of a low-pass filter. However, in the chronic case the rise time is very fast, with a drop in the amplitude of the potential over the pulse width. Part (b) shows the same plot zoomed in to the peaks of the waveforms to highlight the difference between the acute and chronic cases more clearly. (c) This contour plot shows the iso-potential lines in the tissue surrounding the electrode, for an example simulation of the quasi-static $(0 \mathrm{~Hz})$ case, plus the line of measurement for the potential/ distance curves in part (d). 

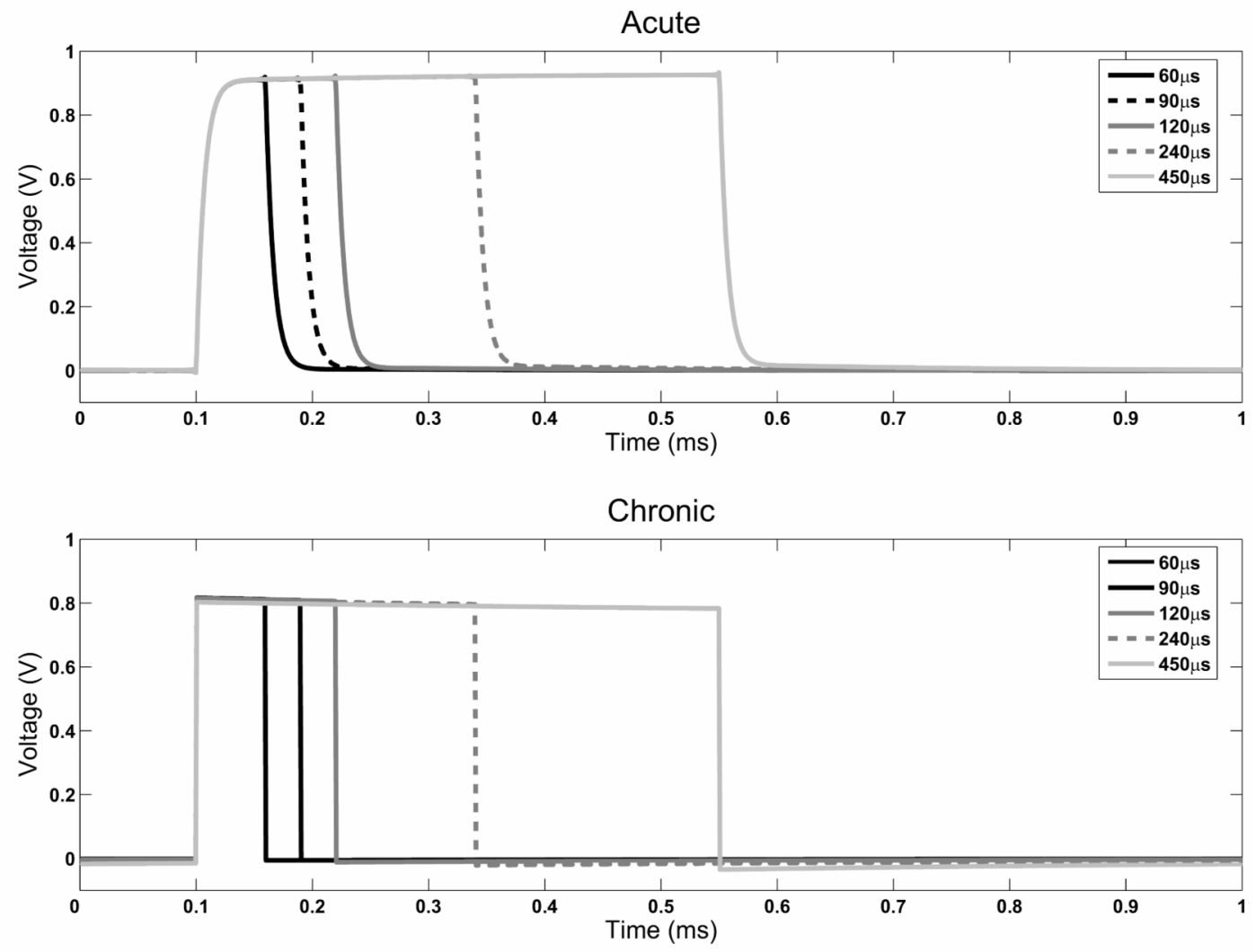

Figure 5.

Comparison of the effect of pulse-width on the induced waveforms. The shape of the distorted square pulse is maintained at all pulse width values in both the acute and chronic simulations. However, the peak amplitude in the acute case increases with increasing pulse width, whereas the drop-off in potential amplitude increases in the chronic case. 

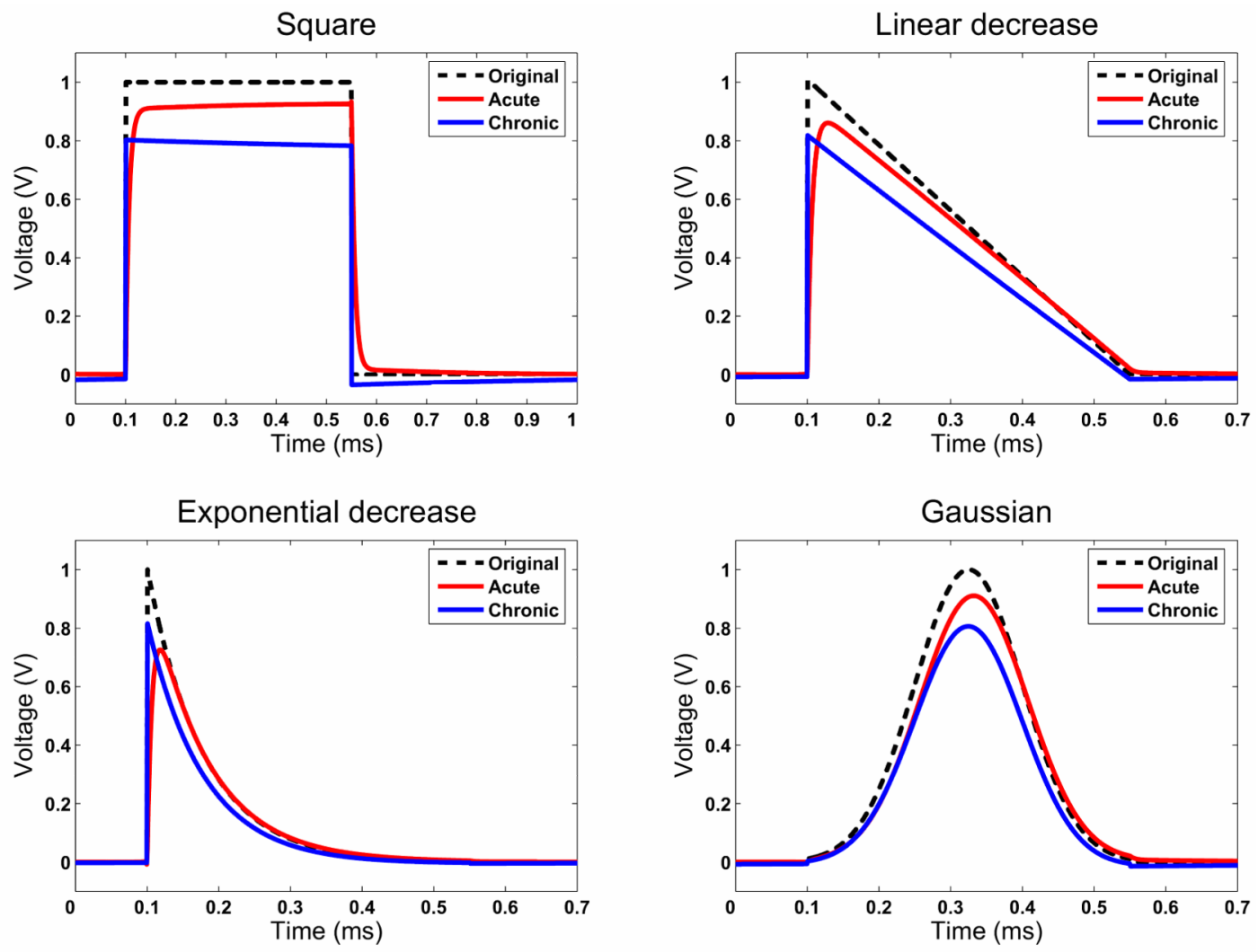

Figure 6.

Comparison of different waveforms of the conventional square pulse, linear decrease, exponential decrease and Gaussian pulses. Less shaping of these types of waveforms manifests compared to the square pulse. Note however, that in the Gaussian case, there appears to be a phase shift of the resulting waveform in the acute case. 


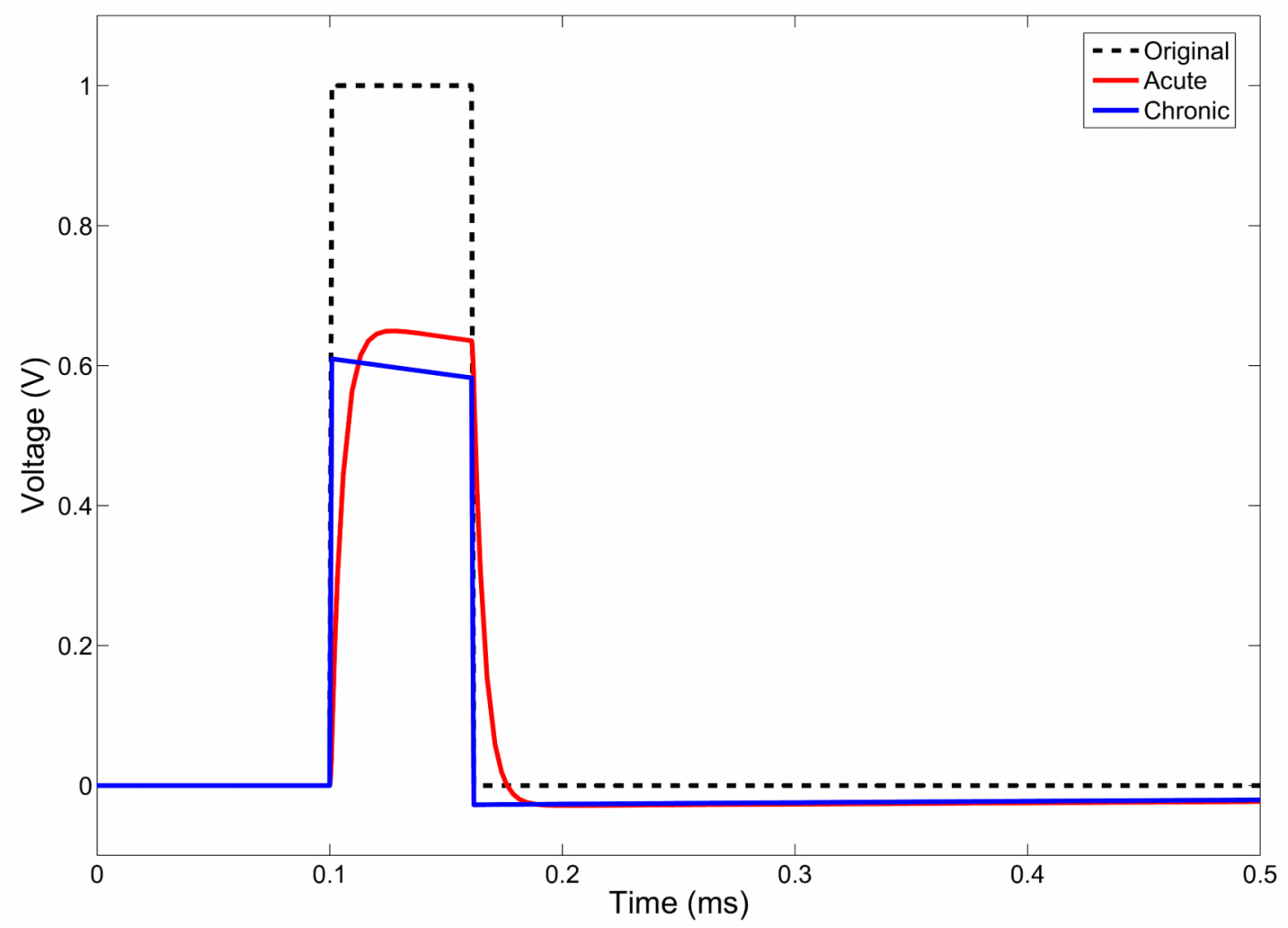

Figure 7.

The waveform shaping in the circuit model. This figure shows the results of the simulation of the circuit model shown in Fig. 3. The resulting waveforms in both the acute and chronic time stages show the same properties as those yielded by the FEM model. 


\section{Table}

Peak amplitude of the waveform induced in the tissue relative to the original waveform, for the square wave and the novel waveforms in both acute and chronic time stages. Also shown is the energy delivered to the tissue, relative to the original square wave, for each waveform

\begin{tabular}{|l|c|c|c|c|}
\hline Waveforms & \multicolumn{2}{|c|}{ Peak amplitude relative to original waveform } & \multicolumn{2}{c|}{ Energy delivered relative to original square pulse } \\
\hline & Acute & Chronic & Acute & Chronic \\
\hline Square & $93 \%$ & $80 \%$ & $93 \%$ & $76 \%$ \\
\hline Linear decrease & $86 \%$ & $82 \%$ & $47 \%$ & $38 \%$ \\
\hline Exponential decrease & $73 \%$ & $82 \%$ & $16 \%$ & $13 \%$ \\
\hline Gaussian & $91 \%$ & $81 \%$ & $39 \%$ & $32 \%$ \\
\hline
\end{tabular}

